\title{
New Ways to Think About CS Education
}

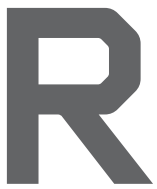

ECENT ARTICLES IN COTmunications have touched on our professional responsibilities to society, including the importance of attracting more and diverse people to computing, integrating ethics into the technology development cycle, and mitigating the effect of our conferences on the environment. Two new approaches could have impact on both the quality of CS education and those societal concerns.

\section{Reinforcing What Students Learn about Ethics}

For decades, accreditation agencies have required that $\mathrm{CS}$ students be exposed to the ethical responsibilities of a professional, typically through a course dedicated to societal aspects of computing. But as technology has taken over more aspects of daily life, concern has grown about whether CS graduates really learn enough about ethics and accountability. Harvard University has come up with a unique solution, called "Embedded EthiCS." It combines two tactics: interspersing ethical discussions throughout the curriculum, and engaging the help of philosophy faculty to co-teach relevant material. The evolution of the initiative was described in the August 2019 issue (p. 54).

Taking an integrative approach reinforces the importance of ethics in creating and applying technology. Teaching ethics at just one point in the curriculum doesn't really convey the extent to which we, as technologists, need to be alert to the ethical aspects of everything we do. The notion of pairing ethical scenarios with different topics should have been obvious long ago; the accompanying table shows examples from common courses in the CS degree curriculum.

Partnering with philosophy experts adds

Courses typically included in the CS curriculum.
\begin{tabular}{ll} 
Course & Types of Ethical Challenges Addressed \\
\hline Networks & Social media, fake news, and the ethics of censorship \\
\hline Programming Languages & Ethics in software verification and validation \\
\hline Data Systems & Privacy in the design of data systems \\
\hline Usability & Inclusive design and equality of opportunity \\
\hline Artificial Intelligence & Machines and moral decision-making \\
\hline
\end{tabular}

another dimension. In computing, we focus on finding the best algorithm to solve a problem, but do not always analyze the impact of solving it in this way. Learning more about the framework of philosophical thinking gives students tools to recognize when they are actually making ethical as well as technical choices - and that failure to consider those consequences may lead to unwise choices. (Learn more at embeddedethics.seas.harvard.edu)

\section{Creating New Pathways into CS}

The shortage of computer scientists is a worldwide problem, but it has proven difficult to create new pathways into computing. Oregon State University tackled the problem by targeting a largely untapped resource: people with degrees from other disciplines who want to move into CS. It allows individuals without any computing background to complete a second degree in CS, on an accelerated schedule (similar to medical schools offering accelerated MD degrees to researchers with a Ph.D. in science) and from their own location.

The goal was attracting new audiences to CS, so a key component is that although it is an accredited BS program, no prior knowledge is assumed. Students come from fields as diverse as music, forestry, and healthcare, with about $25 \%$ already holding advanced degrees (including Ph.D. and MD). Almost half report no prior experience with either program- ming or online studies. Students can pace their coursework if they need to balance study with work or caregiving responsibilities.

In order to retain students, it was necessary to create a virtual environment that mirrors the on-campus experience. Software was developed to mimic the "active learning" format used in campus classrooms, including small-group instruction and interactive sessions with instructors and teaching assistants. Students have the same advising, tutoring, and career services support they would receive on campus, and a new near-peer mentoring program pairs entering students with experienced ones. An active online community has developed where past and current students interact with peers and potential students. (Learn more at eecs.oregonstate.edu/academic/ online-cs-postbacc.)

If adopted widely, both approaches have ancillary benefits that could prove transformative as well. The Harvard initiative keeps ethical issues in the forefront of teachers' minds, too. The Oregon State initiative helps increase the diversity of the computing workforce, and raises awareness of how remote participation can mimic "being there" without the environmental impact of travel or commuting. As recent Communications authors have pointed out, all three of those cultural changes are important to the future of our profession. 